\title{
Non-routine Discharge Disposition is Associated with Post- discharge Complications and 30-day Readmissions Following Craniotomy for Brain Tumor Resection
}

\author{
Nikita Lakomkin, BA ${ }^{1,2}$ and Constantinos G. Hadjipanayis, MD, $\mathrm{PhD}^{1,2}$ \\ ${ }^{1}$ Department of Neurosurgery, Icahn School of Medicine at Mount Sinai, Mount Sinai Health \\ System \\ ${ }^{2}$ Department of Neurosurgery, Icahn School of Medicine, Mount Sinai Beth Israel, Mount Sinai \\ Health System
}

\section{Abstract}

Several studies have reported an association between high-volume brain tumor centers and greater rates of routine discharge disposition in the context of better outcomes. However, the relationship between in-hospital complications, discharge destination, and postoperative adverse events (AEs) remains unexplored. The purpose of this study was thus to use a large, prospectively collected database to examine the association between discharge destination, post-discharge complications, readmissions, and reoperations among patients undergoing craniotomy for brain tumor. The 20112014 National Surgical Quality Improvement (NSQIP) database was employed to identify all adult patients who underwent a craniotomy for brain tumor resection. Demographics, comorbidities, and perioperative variables were collected for each patient. Univariate statistics with subsequent binary logistic regression analyses were used to explore the relationship between these perioperative factors and postoperative events, including major post-discharge complications, minor postdischarge AEs, readmissions, and return to the operating room (ROR). Significant variables such as demographics, comorbidities, operative time, body mass index, ASA classification and predischarge complications were controlled for in each model. Of the 14,854 patients identified, 11,409 (77.9\%) were discharged home. After controlling for comorbidities and in-hospital AEs, discharge to skilled rehabilitation was an independent predictor of major post-discharge complications (OR: 1.74, 95\%CI: 1.31-2.30, p<0.001), minor post-discharge events (OR: 1.60, 95\%CI: $1.07-2.41, \mathrm{p}=0.024$ ), and ROR (OR: 1.68, 95\%CI: 1.27-2.22, $\mathrm{p}<0.001$ ). Discharge to a care facility was predictive of major complications (OR: 1.51, 95\%CI: 1.04-2.19, $\mathrm{p}=0.030$ ) and ROR (OR: 2.02, 95\% CI: 1.46-2.80, $\mathrm{p}<0.001$ ). These factors may be considered in discharge planning and further outcomes studies for patients undergoing resection.

\section{Keywords}

Discharge destination; NSQIP; readmissions; adverse events; morbidity

Correspondence: Constantinos G. Hadjipanayis, MD, PhD, Mount Sinai Beth Israel - Phillips Ambulatory Care Center, 10 Union Square East, Suite 5E, New York, NY 10003, Constantinos.Hadjipanayis@mountsinai.org.

Conflicts of Interest and Source of Funding: None 


\section{INTRODUCTION}

Surgical resection via craniotomy is the standard of care in the treatment of many types of intracranial tumors. However, there is a well-documented risk of transient and permanent neurological deficits as well as other complications following these procedures [1-7]. Certain patients undergoing craniotomy for tumor resection may benefit from closer surveillance and trained assistance during recovery, and as such over $20 \%$ of these patients are discharged to skilled nursing facilities (SNF) or inpatient rehabilitation facilities (IRF) $[1,8]$. However, recent studies evaluating the influence of discharge disposition on postoperative outcomes following various surgical procedures have questioned the positive impact of these centers, particularly in the context of potentially increased risk for postdischarge complications and the high costs associated with therapy [9-11]. In a cohort of brain tumor patients undergoing resection, the median total hospitalization costs were reported to be over $40 \%$ higher for patients discharged to a care facility when compared to those who were discharged home [12]. As such, identification of the perioperative risk factors predictive of non-home discharge disposition may facilitate informed disposition selection and more effective perioperative resource utilization.

Despite several studies reporting an association between in-hospital complications, discharge destination, and post-discharge outcomes in other surgical cohorts [10, 13], these relationships remain relatively unexplored in neurosurgical oncology. Prior studies have highlighted a significant association between higher volume brain tumor centers/providers and greater rates of routine discharge as well as superior outcomes, but the impact of disposition selection on outcomes is not well-established $[1,14,15]$. We hypothesized that non-home discharge disposition is a significant, independent risk factor for post-discharge adverse events. As such, the purpose of this study was to utilize a large, prospectively collected database in order to examine the association between discharge destination, postdischarge complications, readmissions, and return to the operating room among patients undergoing craniotomy for brain tumor.

\section{METHODS}

Data were collected from the 2011-2014 National Surgical Quality Improvement (NSQIP) database. This registry provided a logistically attractive framework via which to test our hypothesis. First, a wide array of variables, including many preoperative comorbidities, laboratory values, and readmission/length of stay (LOS) are manually extracted by dedicated NSQIP coordinators and can be controlled for in the predictive models. The detailed data regarding timing can be utilized to stratify adverse events relative to discharge. Second, the dataset undergoes robust oversight and assessment to ensure data integrity [16].

All adult patients undergoing craniotomy for brain tumor were identified using Current Procedural Terminology (CPT) and International Classification of Diseases (ICD)-9 coding as per prior NSQIP analyses of this patient population [8]. Inclusion criteria consisted of: 1) adult patients over the age of 18,2 ) patients who underwent a craniotomy or craniectomy for tumor excision, as characterized by CPT codes $(61500,61510,61512,61518,61519,61520$, 
61521, 61526, or 61530), and 3) a postoperative ICD-9 diagnosis describing a primary brain tumor, secondary brain tumor, tumor of the meninges, or cranial nerve tumor.

Demographic variables, including age, sex, race, and body mass index (BMI) as well as any preoperative comorbidities were recorded for each patient. Comorbidity variables included diabetes, cigarette use, functional dependency, ventilator use, chronic obstructive pulmonary disease (COPD), hypertension, ascites, congestive heart failure (CHF), myocardial infarction (MI), dyspnea, peripheral vascular disease (PVD), transient ischemic attack (TIA), cerebrovascular accident (CVA), history of renal failure, percutaneous coronary intervention (PCI), bleeding disorder, rapid weight loss, prior transfusion, steroid use, dialysis, and sepsis. Perioperative factors included American Society of Anesthesiologists (ASA) score, operative time, and total hospital LOS. Previously described CPT and ICD-9 coding was employed to stratify patients by procedure type (supratentorial or infratentorial craniotomy) as well as by histologic diagnosis (primary brain, secondary brain, meninges, or cranial nerve tumor) [8].

Preoperative laboratory values were extracted for each patient, and classified as normal or abnormal based on institutional ranges. These included sodium (normal: $135-145 \mathrm{mEq} / \mathrm{L}$ ), blood urea nitrogen (BUN) (normal: 7-20 mg/dL), creatinine (normal: $0.5-1.2 \mathrm{mg} / \mathrm{dL}$ ), albumin (3.4-5.4 g/dL), bilirubin (normal: $0.3-1.9 \mathrm{mg} / \mathrm{dL}$ ), serum glutamic oxaloacetic transaminase (SGOT) (10-34 IU/L), alkaline phosphatase (44-147 IU/L), white blood cell count (WBC) $(4.5-10 \mathrm{k} / \mathrm{mcL})$, hematocrit $(38-54 \%)$, and platelet count $(150-400 \mathrm{k} / \mathrm{mcL})$.

Postoperative events, including discharge destination (home, skilled rehabilitation, or care facility) as well as 30-day readmissions were recorded. Postoperative complications, which are recorded in the registry within 30 days following surgery, were sub-divided into pre- and post-discharge adverse events based on the timing of the occurrence. The main outcome variables in our analysis included post-discharge major complications, post-discharge minor complications, readmissions, and return to the operating room (OR) within 30 days. Major and minor complications were classified as per prior NSQIP studies in the literature [17]. Major complications included prolonged ventilator use of 48 hours or more, unplanned reintubation, sepsis, septic shock, coma, stroke, cardiac arrest, myocardial infarction (MI), venous thromboembolism, infection of the surgical site or organ space, and acute renal failure. Perioperative blood transfusion, pneumonia, urinary tract infection (UTI), wound dehiscence, and renal insufficiency were classified as minor complications.

\section{Statistical Analysis}

Univariate calculations were performed to report the mean and standard deviation (SD) of continuous variables along with the number and proportion of the total for categorical variables. Bivariate analyses were then performed to test the perioperative variables for association with non-home discharge. Variables resulting in $\mathrm{P}<0.1$ were subsequently incorporated into a multivariable, binary logistic regression model in order to identify significant predictors of non-routine discharge disposition. These analyses were then replicated to identify independent predictors of major post-discharge complications, minor post-discharge complications, readmissions, and return to the operating room in order to explore the relationship between discharge destination and subsequent adverse events. 
Separate models were constructed to assess potential risks associated with discharge to skilled rehabilitation or care facility as compared to home discharge. The area under the receiver operating characteristic curve (AUC) was computed to assess the predictive capacity of each model and the Hosmer-Lemeshow test was performed to test for lack of goodnessof-fit to the data. All statistical analyses were performed using IBM SPSS Statistics 22 (IBM, Armonk, NY).

\section{RESULTS}

\section{Case Series Characteristics}

A total of 14,854 patients were included in our study. In this cohort, the mean age was 56 $( \pm 15)$ years, the mean BMI was $28( \pm 8)$, and 7,024 (47.3\%) of patients were male. The most prevalent comorbidities were hypertension, present in $38.6 \%$ of patients, followed by cigarette use in $19.4 \%$ of patients. The majority $(80.4 \%)$ of the tumors were classified as supratentorial. The mean operative time was $214( \pm 134)$ minutes and the mean hospital LOS was $7( \pm 8)$ days. At discharge, $77.9 \%$ of patients were discharged home. These case series characteristics are outlined in Table 1.

The mortality rate in our series was 3.3\%. Prior to discharge, $4.7 \%$ of patients experienced a major complication and $6.7 \%$ experienced a minor complication. The most common predischarge complications were perioperative transfusion (4.8\%), ventilator use for more than 48 hours (2.2\%), and unplanned intubation (1.6\%). Following discharge, $4.8 \%$ of patients experienced a major complication and $1.7 \%$ had a minor complication. The most frequent post-discharge complications were DVT (1.4\%), UTI (1.0\%), and PE (1.0\%). The full breakdown depicting the incidence and timing of these complications can be found in Table 2.

The variables resulting in $\mathrm{P}<0.1$ via bivariate analyses are reported in Table 3 . These factors were then incorporated into the corresponding multivariable regressions for each outcome of interest. There were 8 independent predictors of non-home discharge in the multivariable model. The two modifiable risk factors included hypertension (OR: 1.21, 95\% CI: 1.06-1.39, $\mathrm{p}=0.005)$ and BMI (OR: 1.01, 95\%CI: 1.00-1.02, p=0.039). Preoperative characteristics, including dependent functional status (OR: 2.54, 95\%CI: 2.03-3.18, p<0.001), age (OR: 1.035, 95\%CI: $1.03-1.04, \mathrm{p}<0.001)$, and ASA score $(\mathrm{p}<0.001)$ were also significantly predictive. In addition, pre-discharge complications of stroke (OR: 5.95, 95\%CI: 3.06$11.58, \mathrm{p}<0.001$ ) and transfusion (OR: $1.48,95 \% \mathrm{CI}: 1.14-1.19, \mathrm{p}=0.003$ ) were significantly associated with non-home discharge. Finally, perioperative predictors included operative time (OR: 1.001, 95\%CI: 1.001-1.002, p<0.001) and total hospital stay (OR: 1.12, 95\%CI: $1.11-1.14, \mathrm{p}<0.001)$. The computed AUC for the model was 0.81 . These findings are presented in Table 4.

After controlling for significant comorbidities, perioperative variables, and inhospital adverse events, discharge to skilled rehabilitation remained an independent predictor of major post-discharge complications (OR: 1.74, 95\%CI: 1.31-2.30, p<0.001), minor postdischarge events (OR: 1.60, 95\% CI: 1.07-2.41, $\mathrm{p}=0.024$ ), and return to the operating room (OR: $1.68,95 \%$ CI: $1.27-2.22, \mathrm{p}<0.001$ ). Discharge to a care facility was predictive of major 
complications (OR: $1.51,95 \% \mathrm{CI}: 1.04-2.19, \mathrm{p}=0.030$ ) and return to the operating room (OR: 2.02, 95\%CI: 1.46-2.80, $\mathrm{p}<0.001$ ). The associations between discharge disposition and these post-discharge adverse outcomes, along with the corresponding AUCs of the models, can be found in Table 5. There was no lack of goodness-of-fit to the data.

\section{DISCUSSION}

Several studies have reported that patients undergoing craniotomy for brain tumor resection at high-volume centers experience both significantly fewer complications and reduced rates of non-routine discharge disposition [1, 14]. However, the direct relationship between discharge disposition and postoperative outcomes was not assessed in their analyses. In addition, although approximately $22 \%$ of brain tumor patients are discharged to a care facility following resection, the predictors of non-routine discharge have not been adequately addressed $[8,15]$. In this analysis, we identified the specific perioperative variables and predischarge complications that were risk factors for non-home discharge. These data also demonstrated significant associations between discharge to skilled rehabilitation or care facilities and post-discharge adverse outcomes, such as major complications and return to the operating room, when controlling for other significant factors.

Determining a patient's discharge disposition is one of the major decisions that must be made in the postoperative period [11]. When deemed necessary, the logistics of arranging and facilitating the transfer of a patient to a care facility can lead to delays and unnecessarily extend a patient's hospital stay $[9,18,19]$. Taking proactive steps for patients at higher risk for non-routine discharge disposition has been proposed as a means of streamlining the discharge process [19]. As such, investigators in a variety of surgical subspecialties have sought to develop instruments to predict which patients may be at increased risk for nonroutine discharge $[9,20,21]$. These tools range from simple calculators designed to be used for a wide array of patients [20] to instruments developed for individual procedures, such as primary total joint arthroplasty [21-23]. Identification of the characteristics and perioperative events associated with non-home discharge in specific patient populations can facilitate more effective risk assessment and management [10]. Our model, which incorporated patient demographics and comorbidities, preoperative laboratory lab values, pre-discharge complications, and other perioperative variables, provided strong discriminatory capacity ( $\mathrm{AUC}=0.81$ ). These findings may have utility in the development of readmission risk assessment tools for patients undergoing brain tumor resection.

Although a full analysis of the risks for non-routine discharge disposition following craniotomy for tumor resection has not been performed in the literature, several pertinent factors have been identified as part of studies investigating other clinical phenomena. Trinh et al.'s evaluation of the effect of surgeon and hospital brain tumor case volume on outcomes revealed that, as in our study, traits like older age and higher comorbidity burden decrease the likelihood of routine discharge [15]. However, their analysis, which focused primarily on institutional factors, included a limited number of patient and operative characteristics as covariates and only recorded comorbidities as part of a cumulative score. Additionally, Dasenbrock et al. utilized the NSQIP database to examine drivers of hospital LOS following craniotomy for tumor [8]. They found that greater LOS was significantly associated with 
non-routine discharge, a conclusion that is corroborated by our results. Several of our other findings are supported by similar studies performed for different surgical populations. For instance, age, hypertension, and operative complications, which were identified as predictors of non-home discharge in our study, were significantly associated with discharge destination in a cohort of patients undergoing spinal fusion [24]. Hypertension is a modifiable risk factor, and has been shown to be significantly associated with adverse post-operative events $[25,26]$. Adequate preoperative management via a medication regimen may provide some benefit to these patients.

The relationship between discharge disposition and subsequent outcomes is fairly unexplored, particularly in the neurosurgery literature [10, 13, 27]. A recent analysis of hospital readmissions following brain tumor resection included discharge destination as possible driver, although this relationship did not reach statistical significance upon multivariable analysis [27]. The most comprehensive study of this nature was performed by Keswani et al., who utilized the 2011-2013 NSQIP database to collect data on patients undergoing joint replacement [10]. The authors found that non-routine discharge was significantly associated with unplanned 30-day readmission and severe adverse events. These results corroborate aspects of our findings, where patients discharged to rehabilitation or care facilities experienced greater incidence of major complications, minor complications, and return to the operating room. A variety of factors may contribute to these findings. For one, there are inherent risks to undergoing therapy at these types of facilities that are similar to those associated with inpatient settings. Pathogens like Methicillin-resistant

Staphylococcus aureus (MRSA) can be found in skilled care facilities, and the increased risk of exposure may lead to complications requiring hospital readmission [28, 29]. In addition, substantial heterogeneity in both the quality of care and adherence to established protocols has been identified. A report by the Department of Health and Human Services reviewing a sample of SNF stays, for instance, found significant variability in the establishment of appropriate care, wound treatment, and patient discharge plans [30]. Other studies have assessed the reimbursement mechanisms surrounding SNF stays, which are currently not linked to outcome metrics like hospital readmission [10,31]. However, it is clear that there is a subset of patients who simply cannot be discharged to home following resection. As such, these factors may be considered on a case-by-case basis in patient counseling and discharge planning. Further studies are needed to elucidate the relationship between discharge disposition and the development of adverse events leading to readmission.

These findings must be assessed in the context of limitations in study design. Although the registry data are collected prospectively, this represents a retrospective analysis and is thus at inherent risk for selection bias. Additionally, there are variables that are not recorded in the NSQIP database and thus could not be used in our analysis. First, provider and hospital volume have been shown to be associated with discharge disposition, but could not be examined in the present study. Second, variables specific to neurosurgical oncology, such as biomarker data and tumor recurrence are not collected. Third, socioeconomic variables including family support could not be assessed. As such, it was not possible to determine the rationale behind decisions regarding discharge destination for each patient. Finally, adverse events are only tracked within the first 30-day window, precluding the examination of the relationship between discharge disposition and long-term postoperative outcomes. 


\section{CONCLUSION}

A number of patient characteristics and perioperative factors were shown to be predictive of discharge disposition in this study. Non-routine discharge was significantly associated with an array of complications as well as return to the operating room following hospital discharge. These factors may be considered in discharge planning and further outcomes studies for patients undergoing resection.

\section{References}

1. Barker FG, Curry WT, Carter BS. Surgery for primary supratentorial brain tumors in the United States, 1988 to 2000: The effect of provider caseload and centralization of care. Neuro-Oncol. 2005; 7:49-63. DOI: 10.1215/S1152851704000146 [PubMed: 15701282]

2. Bekelis K, Bakhoum SF, Desai A, et al. Outcome prediction in intracranial tumor surgery: the National Surgical Quality Improvement Program 2005-2010. J Neurooncol. 2013; 113:57-64. DOI: 10.1007/s11060-013-1089-3 [PubMed: 23436132]

3. Cabantog AM, Bernstein M. Complications of first craniotomy for intra-axial brain tumour. Can J Neurol Sci J Can Sci Neurol. 1994; 21:213-218.

4. Cote DJ, Dubois HM, Karhade AV, Smith TR. Venous Thromboembolism in Patients Undergoing Craniotomy for Brain Tumors: A U.S. Nationwide Analysis. Semin Thromb Hemost. 2016; 42:870876. DOI: 10.1055/s-0036-1592306 [PubMed: 27764877]

5. Lonjaret L, Guyonnet M, Berard E, et al. Postoperative complications after craniotomy for brain tumor surgery. Anaesth Crit Care Pain Med. 2016; doi: 10.1016/j.accpm.2016.06.012

6. Mukand JA, Blackinton DD, Crincoli MG, et al. Incidence of neurologic deficits and rehabilitation of patients with brain tumors. Am J Phys Med Rehabil. 2001; 80:346-350. [PubMed: 11327556]

7. Sawaya R, Hammoud M, Schoppa D, et al. Neurosurgical outcomes in a modern series of 400 craniotomies for treatment of parenchymal tumors. Neurosurgery. 1998; 42:1044-1055. 1056. [PubMed: 9588549]

8. Dasenbrock HH, Liu KX, Devine CA, et al. Length of hospital stay after craniotomy for tumor: a National Surgical Quality Improvement Program analysis. Neurosurg Focus. 2015; 39:E12.doi: 10.3171/2015.10.FOCUS15386

9. Barsoum WK, Murray TG, Klika AK, et al. Predicting Patient Discharge Disposition After Total Joint Arthroplasty in the United States. J Arthroplasty. 2010; 25:885-892. DOI: 10.1016/j.arth. 2009.06.022 [PubMed: 19729270]

10. Keswani A, Weiser MC, Shin J, et al. Discharge Destination After Revision Total Joint Arthroplasty: An Analysis of Postdischarge Outcomes and Placement Risk Factors. J Arthroplasty. 2016; 31:1866-1872. e1. DOI: 10.1016/j.arth.2016.02.053 [PubMed: 27172864]

11. London DA, Vilensky S, O'Rourke C, et al. Discharge Disposition After Joint Replacement and the Potential for Cost Savings: Effect of Hospital Policies and Surgeons. J Arthroplasty. 2016; 31:743748. DOI: 10.1016/j.arth.2015.10.014 [PubMed: 26725136]

12. Missios S, Bekelis K. Drivers of hospitalization cost after craniotomy for tumor resection: creation and validation of a predictive model. BMC Health Serv Res. 2015; 15:85.doi: 10.1186/ s12913-015-0742-2 [PubMed: 25756732]

13. Bini SA, Fithian DC, Paxton LW, et al. Does Discharge Disposition After Primary Total Joint Arthroplasty Affect Readmission Rates? J Arthroplasty. 2010; 25:114-117. DOI: 10.1016/j.arth. 2008.11.007 [PubMed: 19150214]

14. Curry WT, McDermott MW, Carter BS, Barker FG. Craniotomy for meningioma in the United States between 1988 and 2000: decreasing rate of mortality and the effect of provider caseload. J Neurosurg. 2005; 102:977-986. DOI: 10.3171/jns.2005.102.6.0977 [PubMed: 16028755]

15. Trinh VT, Davies JM, Berger MS. Surgery for primary supratentorial brain tumors in the United States, 2000-2009: effect of provider and hospital caseload on complication rates. J Neurosurg. 2015; 122:280-296. DOI: 10.3171/2014.9.JNS131648 [PubMed: 25397366] 
16. Belmont PJ, Goodman GP, Kusnezov NA, et al. Postoperative myocardial infarction and cardiac arrest following primary total knee and hip arthroplasty: rates, risk factors, and time of occurrence. J Bone Joint Surg Am. 2014; 96:2025-2031. DOI: 10.2106/JBJS.N.00153 [PubMed: 25520335]

17. Lukasiewicz AM, Grant RA, Basques BA, et al. Patient factors associated with 30-day morbidity, mortality, and length of stay after surgery for subdural hematoma: a study of the American College of Surgeons National Surgical Quality Improvement Program. J Neurosurg. 2016; 124:760-766. DOI: 10.3171/2015.2.JNS142721 [PubMed: 26315000]

18. AlHilli MM, Tran CW, Langstraat CL, et al. Risk-Scoring Model for Prediction of Non-Home Discharge in Epithelial Ovarian Cancer Patients. J Am Coll Surg. 2013; 217:507-515. DOI: 10.1016/j.jamcollsurg.2013.04.036 [PubMed: 23816386]

19. Pattakos G, Johnston DR, Houghtaling PL, et al. Preoperative prediction of non-home discharge: a strategy to reduce resource use after cardiac surgery. J Am Coll Surg. 2012; 214:140-147. DOI: 10.1016/j.jamcollsurg.2011.11.003 [PubMed: 22265219]

20. Hyder JA, Wakeam E, Habermann EB, et al. Derivation and Validation of a Simple Calculator to Predict Home Discharge after Surgery. J Am Coll Surg. 2014; 218:226-236. DOI: 10.1016/ j.jamcollsurg.2013.11.002 [PubMed: 24440065]

21. Oldmeadow LB, McBurney H, Robertson VJ. Predicting risk of extended inpatient rehabilitation after hip or knee arthroplasty. J Arthroplasty. 2003; 18:775-779. [PubMed: 14513453]

22. Hansen VJ, Gromov K, Lebrun LM, et al. Does the Risk Assessment and Prediction Tool predict discharge disposition after joint replacement? Clin Orthop. 2015; 473:597-601. DOI: 10.1007/ s11999-014-3851-z [PubMed: 25106801]

23. Slover J, Mullaly K, Karia R, et al. The use of the Risk Assessment and Prediction Tool in surgical patients in a bundled payment program. Int J Surg. 2017; 38:119-122. DOI: 10.1016/j.ijsu. 2016.12.038 [PubMed: 28034774]

24. Best MJ, Buller LT, Falakassa J, Vecchione D. Risk Factors for Nonroutine Discharge in Patients Undergoing Spinal Fusion for Intervertebral Disc Disorders. Iowa Orthop J. 2015; 35:147-155. [PubMed: 26361458]

25. Eagle KA, Berger PB, Calkins H, et al. ACC/AHA Guideline Update for Perioperative Cardiovascular Evaluation for Noncardiac Surgery-Executive Summary A Report of the American College of Cardiology/American Heart Association Task Force on Practice Guidelines (Committee to Update the 1996 Guidelines on Perioperative Cardiovascular Evaluation for Noncardiac Surgery). Circulation. 2002; 105:1257-1267. [PubMed: 11889023]

26. Foëx P, Sear JW. The surgical hypertensive patient. Contin Educ Anaesth Crit Care Pain. 2004; 4:139-143. DOI: 10.1093/bjaceaccp/mkh039

27. Dasenbrock HH, Yan SC, Smith TR, et al. Readmission After Craniotomy for Tumor: A National Surgical Quality Improvement Program Analysis. Neurosurgery. 2017; 80:551-562. DOI: 10.1093/neuros/nyw062 [PubMed: 28362921]

28. Coll PP, Crabtree BF, O’Connor PJ, Klenzak S. Clinical risk factors for methicillin-resistant Staphylococcus aureus bacteriuria in a skilled-care nursing home. Arch Fam Med. 1994; 3:357360. [PubMed: 8012624]

29. Thomas JC, Bridge J, Waterman S, et al. Transmission and Control of Methicillin-Resistant Staphylococcus aureus in a Skilled Nursing Facility. Infect Control Amp Hosp Epidemiol. 1989; 10:106-110. DOI: $10.2307 / 30105108$

30. Sollins HL. Office of inspector general report finds skilled nursing facilities "often" fail to meet care planning and discharge planning requirements. Geriatr Nurs N Y N. 2013; 34:233-234. DOI: 10.1016/j.gerinurse.2013.04.005

31. Mor V, Intrator O, Feng Z, Grabowski DC. The Revolving Door Of Rehospitalization From Skilled Nursing Facilities. Health Aff (Millwood). 2010; 29:57-64. DOI: 10.1377/hlthaff.2009.0629 [PubMed: 20048361] 


\section{Table 1}

\section{Case Series Characteristics}

\begin{tabular}{|c|c|}
\hline Demographics & $\mathrm{N}=\mathbf{1 4 , 8 5 4}$ \\
\hline Age (mean +/- SD) & $56 \pm 15$ years \\
\hline BMI (mean +/- SD) & $28 \pm 8$ \\
\hline $\operatorname{Sex}(M: F)$ & $7,024: 7,827$ \\
\hline Functionally dependent & $723(4.9 \%)$ \\
\hline \multicolumn{2}{|l|}{ Select Comorbidities } \\
\hline Hypertension & $5,739(38.6 \%)$ \\
\hline Cigarette use & $2,887(19.4 \%)$ \\
\hline Preoperative steroid use & $2,420(16.3 \%)$ \\
\hline Diabetes & $1,689(11.4 \%)$ \\
\hline \multicolumn{2}{|l|}{ Tumor Histology } \\
\hline Primary brain tumor - benign & $599(4.1 \%)$ \\
\hline Primary brain tumor - malignant & $5,314(36.7 \%)$ \\
\hline Primary brain tumor - unknown malignancy & $1242(8.6 \%)$ \\
\hline Secondary brain or extracranial tumor & $3,100(21.4 \%)$ \\
\hline Meninges tumor - benign & $3,262(22.5 \%)$ \\
\hline Meninges tumor - malignant & $120(0.8 \%)$ \\
\hline Cranial nerve - benign & $549(3.8 \%)$ \\
\hline Cranial nerve - malignant & $37(0.2 \%)$ \\
\hline \multicolumn{2}{|l|}{ Tumor Location } \\
\hline Supratentorial & $11,946(80.4 \%)$ \\
\hline Infratentorial & $2,908(19.6 \%)$ \\
\hline \multicolumn{2}{|l|}{ Perioperative Variables } \\
\hline Operative time (mean $+/-\mathrm{SD})$ & $214 \pm 134 \mathrm{~min}$ \\
\hline Hospital length of stay & $7 \pm 8$ days \\
\hline ASA score $1-2$ & $4,149(27.9 \%)$ \\
\hline ASA score 3 & $8,735(58.8 \%)$ \\
\hline ASA score 4-5 & $1,829(12.3 \%)$ \\
\hline Readmitted to hospital & $1,449(9.8 \%)$ \\
\hline Died & $483(3.3 \%)$ \\
\hline \multicolumn{2}{|l|}{ Discharge Destination } \\
\hline Home & $11,409(77.9 \%)$ \\
\hline Skilled rehabilitation & $2,048(14.0 \%)$ \\
\hline Care facility & $1,187(8.1 \%)$ \\
\hline
\end{tabular}

ASA: American Society of Anesthesiologists

BMI: Body Mass Index 


\section{Table 2}

Incidence of the Most Common Postoperative Complications

\begin{tabular}{ll}
\hline Before Discharge & \\
\hline Major Complications & $705(4.7 \%)$ \\
Ventilator (>48 hours) & $320(2.2 \%)$ \\
Unplanned intubation & $236(1.6 \%)$ \\
Stroke & $146(1.0 \%)$ \\
DVT & $128(0.9 \%)$ \\
Minor Complications & $998(6.7 \%)$ \\
Perioperative transfusion & $712(4.8 \%)$ \\
UTI & $182(1.2 \%)$ \\
Pneumonia & $181(1.2 \%)$ \\
After Discharge & \\
\hline Major Complications & $712(4.8 \%)$ \\
DVT & $214(1.4 \%)$ \\
PE & $144(1.0 \%)$ \\
Organ space infection & $110(0.7 \%)$ \\
Sepsis & $108(0.7 \%)$ \\
Minor Complications & $258(1.7 \%)$ \\
UTI & $153(1.0 \%)$ \\
Pneumonia & $81(0.5 \%)$ \\
\hline &
\end{tabular}

DVT: Deep vein thrombosis

PE: Pulmonary embolism

UTI: Urinary tract infection 
Table 3

Variables Resulting in $\mathrm{P}<0.1$ via Bivariate Analysis With Outcome Metrics of Interest

\begin{tabular}{|c|c|}
\hline Non-home Discharge & OR $(95 \% \mathrm{CI})$ \\
\hline Age & $1.041(1.038-1.044)$ \\
\hline BMI & $0.997(0.993-1.002)$ \\
\hline Male sex & $1.02(0.95-1.10)$ \\
\hline Diabetes & $2.17(1.94-2.42)$ \\
\hline Cigarette use & $0.89(0.81-0.99)$ \\
\hline Dyspnea & $1.35(1.14-1.61)$ \\
\hline Dependent functional status & $4.56(3.90-5.34)$ \\
\hline COPD & $1.45(1.22-1.72)$ \\
\hline Hypertension & $2.03(1.87-2.19)$ \\
\hline Renal failure & $8.84(2.77-28.21)$ \\
\hline Dialysis & $2.75(1.37-5.54)$ \\
\hline Steroid use & $1.13(1.02-1.25)$ \\
\hline Weight loss & $1.86(1.48-2.34)$ \\
\hline Bleeding disorder & $2.21(1.75-2.78)$ \\
\hline Superficial SSI before discharge & $4.55(1.69-12.21)$ \\
\hline Organ space SSI before discharge & $5.15(2.39-11.11)$ \\
\hline PE before discharge & $4.68(2.69-8.16)$ \\
\hline Progressive renal insufficiency before discharge & $3.53(0.88-14.12)$ \\
\hline Acute renal failure before discharge & $1.001(1.000-1.002)$ \\
\hline UTI before discharge & $7.54(5.48-10.37)$ \\
\hline Stroke before discharge & $16.45(10.45-25.88)$ \\
\hline Cardiac arrest before discharge & $3.93(1.60-9.68)$ \\
\hline MI before discharge & $5.30(1.89-14.91)$ \\
\hline Bleeding transfusion before discharge & $2.81(2.40-3.29)$ \\
\hline DVT before discharge & $8.74(5.82-13.14)$ \\
\hline Sepsis before discharge & $8.00(5.03-12.72)$ \\
\hline Septic shock before discharge & $21.32(7.39-61.48)$ \\
\hline Pneumonia before discharge & $11.44(7.81-16.77)$ \\
\hline Abnormal sodium & $1.55(1.38-1.75)$ \\
\hline Abnormal BUN & $1.75(1.61-1.91)$ \\
\hline Abnormal creatinine & $1.65(1.45-1.88)$ \\
\hline Abnormal albumin & $2.13(1.85-2.44)$ \\
\hline Abnormal WBC & $1.23(1.13-1.33)$ \\
\hline Abnormal hematocrit & $1.54(1.41-1.67)$ \\
\hline Operation time & $1.001(1.001-1.001)$ \\
\hline Total hospital stay & $1.15(1.14-1.16)$ \\
\hline
\end{tabular}

J Neurooncol. Author manuscript; available in PMC 2019 February 01. 


\begin{tabular}{|c|c|c|c|}
\hline \multicolumn{2}{|c|}{ Non-home Discharge } & \multicolumn{2}{|c|}{ OR $(95 \% \mathrm{CI})$} \\
\hline \multicolumn{2}{|c|}{ ASA Score $\Psi$} & \multicolumn{2}{|l|}{ N/A } \\
\hline Major Post-discharge Complications & OR $(95 \% \mathrm{CI}) 6$ & Minor Post-discharge Complications & OR $(95 \% \mathrm{CI})$ \\
\hline Age & $1.011(1.005-1.016)$ & Age & $1.03(1.02-1.04)$ \\
\hline BMI & $1.02(1.01-1.03)$ & BMI & $1.01(1.00-1.03)$ \\
\hline Male sex & $1.28(1.10-1.49)$ & Male sex & $1.00(0.781-1.28)$ \\
\hline Cigarette use & $0.80(0.65-0.98)$ & Diabetes & $1.90(1.39-2.60)$ \\
\hline Dyspnea & $1.53(1.14-2.07)$ & Dyspnea & $2.02(1.31-3.12)$ \\
\hline Dependent functional status & $1.49(1.10-2.01)$ & Dependent functional status & $2.56(1.72-3.79)$ \\
\hline COPD & $1.67(1.25-2.24)$ & COPD & $2.78(1.89-4.11)$ \\
\hline Ascites & $7.95(1.54-41.07)$ & Renal failure & $15.58(4.32-56.2)$ \\
\hline Hypertension & $1.27(1.09-1.48)$ & Hypertension & $2.03(1.59-2.60)$ \\
\hline Steroid use & $1.58(1.32-1.89)$ & Steroid use & $1.83(1.38-2.42)$ \\
\hline Bleeding disorder & $1.78(1.18-2.67)$ & Weight loss & $2.45(1.41-4.24)$ \\
\hline Abnormal sodium & $1.32(1.05-1.66)$ & Abnormal sodium & $1.63(1.15-2.29)$ \\
\hline Abnormal BUN & $1.49(1.26-1.75)$ & Abnormal BUN & $1.65(1.27-2.13)$ \\
\hline Abnormal albumin & $1.08(0.82-1.43)$ & Abnormal albumin & $1.47(0.98-2.20)$ \\
\hline Abnormal WBC & $1.30(1.12-1.52)$ & Abnormal WBC & $1.60(1.25-2.05)$ \\
\hline Bleeding transfusion before discharge & $1.57(1.17-2.11)$ & Abnormal hematocrit & $1.42(1.09-1.84)$ \\
\hline Operation time & $1.000(1.000-1.001)$ & Abnormal platelet count & $2.07(1.51-2.83)$ \\
\hline Total hospital stay & $0.998(0.988-1.008)$ & UTI before discharge & $0.988(0.986-0.989)$ \\
\hline \multirow[t]{6}{*}{ ASA Score } & N/A & Stroke before discharge & $2.46(1.07-5.61)$ \\
\hline & & Bleeding transfusion before discharge & $1.50(0.92-2.44)$ \\
\hline & & DVT before discharge & $4.39(2.21-8.75)$ \\
\hline & & Operation time & $1.000(0.999-1.001)$ \\
\hline & & Total hospital stay & $1.01(1.00-1.03)$ \\
\hline & & ASA Score & N/A \\
\hline Readmission & OR $(95 \% \mathrm{CI})$ & Return to OR & OR $(95 \% \mathrm{CI})$ \\
\hline Age & $1.004(1.000-1.008)$ & BMI & \\
\hline BMI & $1.015(1.008-1.022)$ & Male sex & $1.26(1.09-1.46)$ \\
\hline Male sex & $1.25(1.12-.39)$ & Dyspnea & \\
\hline Diabetes & $1.22(1.04-1.44)$ & Dependent functional status & $1.95(1.50-2.53)$ \\
\hline Dependent functional status & $1.53(1.23-1.90)$ & Hypertension & $1.33(1.15-1.54)$ \\
\hline COPD & $1.27(1.00-1.61)$ & Steroid use & $1.29(1.08-1.55)$ \\
\hline Ascites & $6.90(1.54-30.86)$ & Weight loss & $1.56(1.04-2.32)$ \\
\hline $\mathrm{CHF}$ & $2.60(1.24-5.44)$ & Bleeding disorder & $1.77(1.19-2.62)$ \\
\hline Renal failure & & Abnormal sodium & $1.33(1.07-1.65)$ \\
\hline Hypertension & $1.34(1.20-1.49)$ & Abnormal BUN & $1.17(0.99-1.37)$ \\
\hline Steroid use & $1.39(1.21-1.59)$ & Abnormal albumin & $1.34(1.04-1.74)$ \\
\hline Bleeding disorder & $1.80(1.33-2.44)$ & Abnormal WBC & $1.36(1.17-1.57)$ \\
\hline
\end{tabular}




\begin{tabular}{|c|c|c|c|}
\hline \multicolumn{2}{|c|}{ Non-home Discharge } & \multicolumn{2}{|c|}{ OR $(95 \% \mathrm{CI})$} \\
\hline Prior transfusion & $2.31(1.22-4.35)$ & Abnormal platelet count & $1.22(0.98-1.52)$ \\
\hline Abnormal sodium & $1.31(1.11-1.54)$ & Superficial SSI before discharge & $8.40(2.90-24.23)$ \\
\hline Abnormal BUN & $1.24(1.10-1.39)$ & Deep SSI before discharge & $64.86(13.45-312.76)$ \\
\hline Abnormal creatinine & $1.33(1.11-1.59)$ & Organ space SSI before discharge & $28.19(13.53-58.73)$ \\
\hline Abnormal albumin & $1.19(0.98-1.45)$ & Dehiscence before discharge & $27.65(4.61-165.74)$ \\
\hline Abnormal WBC & $1.18(1.06-1.32)$ & Pneumonia before discharge & $5.08(3.53-7.33)$ \\
\hline Abnormal platelet count & $1.33(1.13-1.56)$ & Intubation before discharge & $10.40(7.86-13.76)$ \\
\hline Pneumonia before discharge & $0.54(0.28-1.02)$ & PE before discharge & $5.33(2.93-9.69)$ \\
\hline Intubation before discharge & $1.43(0.97-2.09)$ & $\begin{array}{l}\text { Progressive renal insufficiency before } \\
\text { discharge }\end{array}$ & $4.58(0.97-21.58)$ \\
\hline Ventilator before discharge & $87.33(26.52-287.62)$ & UTI before discharge & $3.28(2.17-4.97)$ \\
\hline $\begin{array}{l}\text { Progressive renal insufficiency before } \\
\text { discharge }\end{array}$ & $6.14(1.73-21.77)$ & Stroke before discharge & $6.50(4.44-9.51)$ \\
\hline Coma before discharge & $6.13(1.02-36.69)$ & Coma before discharge & $12.27(2.05-73.56)$ \\
\hline Cardiac arrest before discharge & $2.30(0.86-6.14)$ & Cardiac arrest before discharge & $4.62(1.73-12.33)$ \\
\hline Bleeding transfusion before discharge & $1.29(1.02-1.63)$ & MI before discharge & $6.15(1.98-19.11)$ \\
\hline \multirow[t]{8}{*}{ ASA Score } & N/A & Bleeding transfusion before discharge & $2.60(2.04-3.31)$ \\
\hline & & DVT before discharge & $7.49(5.06-11.09)$ \\
\hline & & Sepsis before discharge & $6.09(3.74-9.90)$ \\
\hline & & Septic shock before discharge & $5.38(2.74-10.57)$ \\
\hline & & Ventilator before discharge & $9.3(7.25-11.94)$ \\
\hline & & Operation time & $1.002(1.002-1.002)$ \\
\hline & & Total hospital stay & $1.06(1.05-1.07)$ \\
\hline & & ASA Score & N/A \\
\hline
\end{tabular}

$\Psi$

ASA Score was assessed as part of a $4 \times 2$ table and the OR could thus not be computed

ASA: American Society of Anesthesiologists

BMI: Body Mass Index

BUN: Blood urea nitrogen

CHF: Congestive heart failure

COPD: Chronic obstructive pulmonary disease

DVT: Deep vein thrombosis

PE: Pulmonary embolism

SSI: Surgical site infection

UTI: Urinary tract infection

WBC: White blood cell count 
Table 4

Significant Predictors of Non-home Discharge Disposition $(\mathrm{AUC}=0.81)$

\begin{tabular}{cccc}
\hline Perioperative Risk Factor & Odds Ratio & 95\% Confidence Interval & P \\
\hline Dependent functional status & 2.54 & $2.03-3.18$ & $<0.001$ \\
Hypertension & 1.21 & $1.06-1.39$ & 0.005 \\
Stroke before discharge & 5.95 & $3.06-11.58$ & $<0.001$ \\
Transfusion before discharge & 1.48 & $1.14-1.91$ & 0.003 \\
Age & 1.035 & $1.03-1.04$ & $<0.001$ \\
BMI & 1.01 & $1.00-1.02$ & 0.039 \\
ASA Score & - & - & $<0.001$ \\
Operation time & 1.001 & $1.001-1.002$ & $<0.001$ \\
Hospital length of stay & 1.12 & $1.11-1.14$ & $<0.001$ \\
\hline
\end{tabular}

ASA: American Society of Anesthesiologists

BMI: Body Mass Index 
Table 5

Discharge Disposition as a Predictor of Post-discharge Complications

\begin{tabular}{|c|c|c|c|c|}
\hline Outcome Variable & Odds Ratio & $95 \%$ Confidence Interval & $\mathbf{P}$ & $\mathbf{A U C}$ \\
\hline \multicolumn{5}{|l|}{ Major Complication } \\
\hline Skilled rehabilitation $\Psi$ & 1.74 & $1.31-2.30$ & $<0.001$ & 0.604 \\
\hline Care facility $\Psi$ & 1.51 & $1.04-2.19$ & 0.030 & 0.607 \\
\hline \multicolumn{5}{|l|}{ Minor Complication } \\
\hline Skilled rehabilitation & 1.60 & $1.07-2.41$ & 0.024 & 0.713 \\
\hline Care facility & - & - & - & - \\
\hline \multicolumn{5}{|l|}{ Readmission } \\
\hline Skilled rehabilitation & - & - & - & - \\
\hline Care facility & - & - & - & - \\
\hline \multicolumn{5}{|l|}{ Return to $O R$} \\
\hline Skilled rehabilitation & 1.68 & $1.27-2.22$ & $<0.001$ & 0.688 \\
\hline Care facility & 2.02 & $1.46-2.80$ & $<0.001$ & 0.680 \\
\hline
\end{tabular}

J Neurooncol. Author manuscript; available in PMC 2019 February 01. 